\title{
PaMiNI-Derived Co-Activation Patterns Indicate Differential Hierarchical Levels for Two Ventral Visual Areas of the Fusiform Gyrus
}

\section{PaMiNI basierte Koaktivierungsmuster zeigen unterschiedliche hierarchische Stufen von zwei ventralen visuellen Arealen des Gyrus fusiformis}

Authors

Affiliations
J. Caspers ${ }^{1}$, S. B. Eickhoff ${ }^{2}$, K. Amunts ${ }^{3}$, G. Antoch ${ }^{1}$, K. Zilles ${ }^{4}$

Department for Diagnostic and Interventional Radiology, Universitiy Hospital Düsseldorf, Germany

Institute of Clinical Neuroscience and Medical Psychology, Heinrich-Heine-University Düsseldorf, Germany

C. and O. Vogt Institute for Brain Research, Heinrich-Heine-University Düsseldorf, Germany

Institute of Neuroscience and Medicine (INM-1), Research Centre Jülich, Germany

\section{Key words}

$\checkmark$ pattern mining in neuroimaging

- brainmap database

- coordinate-based meta-analysis

- ventral visual cortex

- fusiform face area

visual word-form area

This article was awarded with the Promotionspreis 2015 of the German Röntgen Society

received $\quad 2.7 .2015$

accepted $\quad 5.8 .2015$

Bibliography

DOI http://dx.doi.org/

10.1055/s-0041-105062

Published online: 2015

Fortschr Röntgenstr 2015; 187 : 892-898 @ Georg Thieme Verlag KG Stuttgart - New York . ISSN 1438-9029

\section{Correspondence}

\section{Herr Dr. Julian Caspers}

Department for Diagnostic and Interventional Radiology,

Universitiy Hospital Düsseldorf Moorenstr. 5

40225 Düsseldorf

Germany

Tel.: ++ 49/(0)211/8117752

Fax: ++49/(0)211/8116145 Julian.Caspers@med.uni-

duesseldorf.de

\section{Zusammenfassung}

$\nabla$

Ziel: Untersuchung der Verteilung von Koaktivierungsmustern der kürzlich entdeckten ventralen visuellen Areale FG1 und FG2 auf dem posterioren Gyrus fusiformis mit dem neuartigen meta-analytischen Verfahren PaMiNI (Pattern Mining in Neurolmaging).

Material und Methoden: Alle Neurobildgebungsstudien, die Aktivierungen in FG1 oder FG2 berichten, wurden aus der BrainMap Datenbank abgerufen. Die berichteten stereotaktischen Aktivierungsfoci im standardisierten Referenzraum wurden mit PaMiNI analysiert. Hierbei wurde Gaussian mixture modeling auf die dreidimensionalen Koordinaten der Aktivierungen angewandt, um die dem entsprechenden Datensatz zugrunde liegenden Hirnregionen zu identifizieren. Anschließend wurde eine Assoziationsanalyse durchgeführt, welche häufige Koaktivierungsmuster zwischen den modellierten Hirnregionen erkennen lässt.

Ergebnisse: Die Koaktivierungsmuster von FG1 waren hauptsächlich innerhalb des visuellen Systems zu finden, insbesondere in frühen visuellen Arealen, und sie waren symmetrisch bezüglich der beiden Hemisphären. FG2 wies zahlreiche extra-visuelle Koaktivierungen auf, vor allem zu interioren frontalen, prämotorischen und parietalen Regionen. Zudem zeigten die Koaktivierungen von FG2 eine klare Lateralisierung zur linken Seite.

Schlussfolgerung: In der Hierarchie des ventralen visuellen Systems zeigt FG1 Merkmale eines intermediären visuellen Areals zwischen den frühen visuellen und den Kategorie-spezifischen höheren Arealen. Die Koaktivierungen von FG2 weisen darauf hin, dass FG2 ein hierarchisch höheres Areal ist, welches wahrscheinlich der posterioren „fusiform face area“ und anteilig der „visual word-form area" entspricht.

\section{Abstract \\ V}

Purpose: To investigate the distribution of co-activation patterns of the recently identified ventral visual areas FG1 and FG2 of the posterior fusiform gyrus using the novel meta-analytic approach PaMiNI (Pattern Mining in Neurolmaging).

Materials and Methods: All neuroimaging experiments reporting activation foci within FG1 or FG2 were retrieved from the BrainMap database. The stereotaxic activation foci in standard reference space were analyzed with PaMiNI. Here, Gaussian mixture modeling was applied to the stereotaxic coordinates of all foci to identify the underlying brain regions of each dataset. Then, association analysis was performed to reveal frequent co-activations across the modeled brain regions.

Results: Co-activation patterns of FG1 were mainly found within the visual system, i. e. in early visual areas, and were symmetrically distributed across both hemispheres. FG2 features several extra-visual co-activations, mainly to inferior frontal, premotor and parietal regions. Furthermore, the co-activations of FG2 showed clear lateralization to the left FG2.

Conclusion: FG1 shows characteristics of an intermediate visual area between the early ventral visual cortex and the category-specific higher-order areas. Co-activation patterns of FG2 indicate that FG2 is a higher-order visual area that probably corresponds to the posterior fusiform face area and partly the visual word-form area.

Key points

- Co-activation patterns of areas FG1 and FG2 were analyzed with PaMiNI.

- FG1 features mainly symmetric co-activations to areas of the visual system.

- FG2 shows several extra-visual co-activations, which are left-lateralized.

- FG1 corresponds to a hierarchically intermediate, FG2 to a higher-order visual area. 
Kernaussagen

- Koaktivivierungsmuster von FG1 und FG2 wurden mit PaMiNI analysiert.

- FG1 weist weitgehend symmetrische Koaktivierungen mit Arealen des visuellen Systems auf.

- FG2 zeigt zahlreiche extra-visuelle Koaktivierungen, die linkslateralisiert sind.

- FG1 entspricht einem hierarchisch intermediären, FG2 einem höhergeordneten visuellen Areal.

- Der PaMiNI Ansatz wird um die Seed-spezifische Kartierung von Koaktivierungsmustern erweitert.
- The PaMiNI approach is extended to seed-specific mapping of co-activation patterns.

Citation Format:

- Caspers J, Eickhoff SB, Amunts K et al. PaMiNI-Derived Co-Activation Patterns Indicate Differential Hierarchical Levels for Two Ventral Visual Areas of the Fusiform Gyrus. Fortschr Röntgenstr 2015; 187: 892-898

\section{Introduction}

$\nabla$

The human ventral visual cortex facilitates its complex function of visual object recognition by processing the visual information in a series of hierarchically arranged stages. This hierarchy is implemented in structurally and functionally distinct brain areas of the ventral visual cortex in a posterior-to-anterior direction, where the most occipital areas represent the unimodal information close to the visual input. More global and invariant representations emerge consecutively in the more rostral areas of the ventral occipitotemporal cortex [1]. The early ventral visual cortex of the occipital lobe comprises areas V1, V2v, V3v and hV4 ( $\bullet$ Fig. 1), which show a retinotopic organization, meaning that neighboring points of the visual field are represented in adjacent groups of neurons. These areas preprocess the visual information for later higher visual areas and enable the detection of contours, simple shapes and color [2]. The higher-order visual cortex in the ventral temporal lobe includes a variety of highly specialized functional areas that facilitate the categorization and recognition of specific objects [3], e.g. the fusiform face area for the recognition of faces, the extrastriate body area for body parts, the parahippocampal place area for houses and places, or the visual word-form area for written language.

We recently identified two hitherto unknown cytoarchitectonic areas, FG1 and FG2, on the posterior fusiform gyrus, which are situated at the junction between the early ventral visual cortex and the higher-order visual regions [4] ( $\bullet$ Fig. 1). FG1 lies anteriorly adjacent to area hV4 on the medial bank of the fusiform gyrus. FG2 borders FG1 laterally and is located on the lateral bank of the fusiform gyrus. An analysis of the receptor architecture of both areas in comparison with other visual and extra-visual areas provided first hints regarding a differential hierarchical position of both areas, i.e. that FG1 represents an intermediate stage between early and higher-order visual areas, while FG2 already belongs to the hierarchically higher-order areas [5]. Furthermore, when analyzing the involvement of both areas in functional neuroimaging experiments and their co-activation patterns [6], FG1 is associated with object recognition on a rather basal level, while FG2 shows high and hemisphere-dependent involvement in face recognition and reading, and thus probably corresponds to the posterior part of the fusiform face area [7] and aspects of the visual word-form area [8].

However, the distribution of actual within-experiment co-activations of FG1 and FG2 is not clear yet. In order to further characterize both areas regarding their interaction with other brain regions, we investigate here their co-activation patterns from functional neuroimaging studies using a recent meta-analytic approach, named PaMiNI ("Pattern Mining in NeuroImaging") [9]. PaMiNI is a novel technique for the identification of frequent co-activation patterns from the large number of published neuroimaging studies based on their reported coordinates in a globally used standard stereotaxic space. PaMiNI utilizes a combination of two well-established data-mining techniques and overcomes several limitations of previous coordinate-based meta-analytic approaches allowing for investigations of the subnetwork structure of functionally connected regions.

In the current study, we perform a PaMiNI analysis on datasets of all functional neuroimaging experiments from the BrainMap database (www.brainmap.org [10]) that report activation within FG1 and FG2, respectively. As the function of an area is strongly related to its microstructural, i.e. cytoarchitectonical organization and its connectivity to other regions $[11,12]$, we specifically test the hypothesis that FG1 and FG2 exhibit differential co-acti-

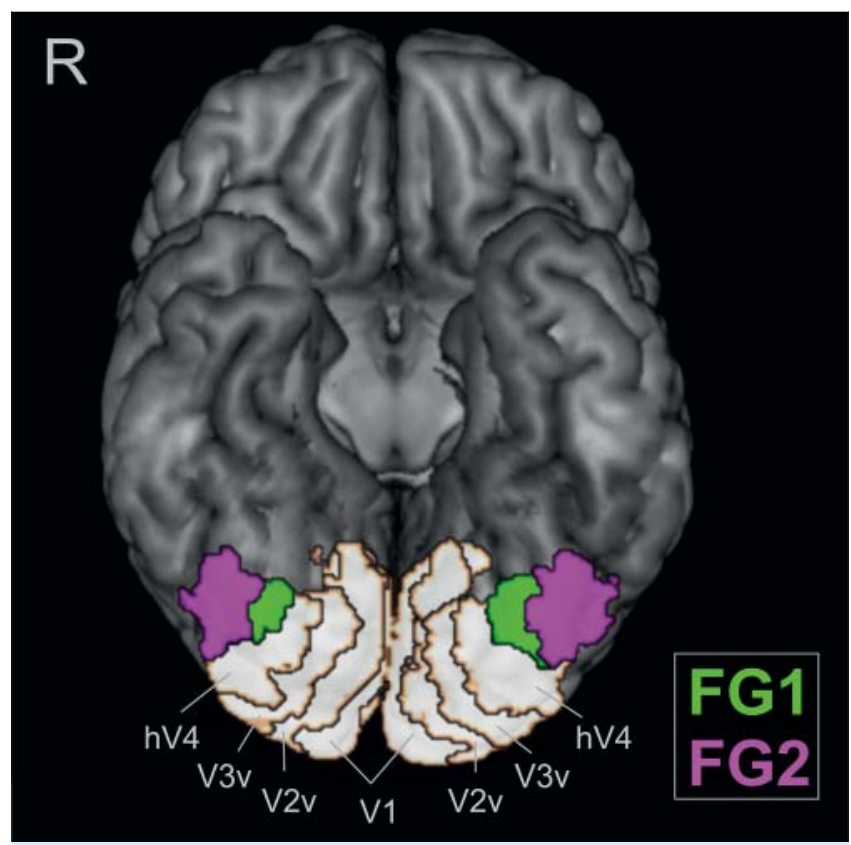

Fig. 1 Cytoarchitectonic areas FG1 (green) and FG2 (violet) of the posterior fusiform gyrus together with early ventral visual areas projected onto the MNI single subject reference brain without cerebellum. Early visual areas V1, V2v [21], V3v and hV4 [19] are delineated in gray. The basal view is shown.

Abb. 1 Zytoarchitektonische Areale FG1 (grün) und FG2 (violett) des posterioren Gyrus fusiformis zusammen mit frühen ventralen visuellen Arealen projiziert auf das MNI Einzelprobanden-Referenzgehirn ohne Cerebellum. Die frühen visuellen Areale V1, V2v [21], V3v und hV4 [19] sind in grau abgegrenzt. Eine Ansicht von basal ist abgebildet. 
vation patterns that make it possible to draw inferences about their hierarchical level in the ventral visual processing stream.

\section{Materials and Methods}

\section{Seed definition and retrieval of neuroimaging experiments}

For the current analysis, we used the same sets of experiments as in our previous analysis of the functional involvement and connectivity of FG1 and FG2 [6]. Here, we identified all neuroimaging experiments from the BrainMap database (www.brainmap.org [10]) that report activation within either area. We employed the maximum probability map representations of FG1 and FG2 derived from observer-independent cytoarchitectonic mapping [4] as seed regions. For each seed, all experiments of healthy subjects in the BrainMap database that featured at least one activation focus within the respective seed, i. e. cytoarchitectonically defined cortical area, were retrieved. The identification of experiments was solely based on their reported location in stereotaxic space (MNI or Talairach). This retrieval resulted in 221 experiments showing activation foci in FG1 and 352 experiments yielding activation within FG2. For the analysis of co-activation patterns, all reported activation foci (i.e. inside and outside the seed region) from each experiment were used.

\section{PaMiNl: Pattern Mining in Neurolmaging}

A detailed description of the PaMiNI method can be found in [9] and [13]. PaMiNI ("Pattern Mining in NeuroImaging") enables the identification of frequent co-activation patterns from the stereotaxic coordinates in a set of neuroimaging experiments by a twostep procedure based on established data-mining techniques. It first models the brain regions underlying the respective dataset from the reported activation foci, and then, in a second step, reveals the frequent co-activations between these modeled brain regions across all experiments.

The modeling of the underlying brain regions in the first step of PaMiNI is facilitated by Gaussian mixture modeling. This is the modeling of $\mathrm{K}_{\mathrm{opt}}$ three-dimensional Gaussian distributions to optimally fit the spatial allocation of the activation foci of all experiments in the dataset, where $\mathrm{K}_{\text {opt }}$ denotes the number of Gaussians. For this, each activation focus is considered as an instance randomly drawn from a mixture of $\mathrm{K}_{\mathrm{opt}}$ three-dimensional Gaussian distributions and the parameters of this mixture (i.e. the mean and covariance matrices of each Gaussian as well as its relative proportion in the mixture) are estimated by optimization of the log-likelihood (LL) function using the expectation-maximization (EM) algorithm [14]. The EM algorithm optimizes the mixture parameters by repeatedly assigning the foci to the Gaussians of the mixture based on the current parameter estimates (expectation) and re-calculating the parameters based on these new assignments (maximization). The resulting fitted $\mathrm{K}_{\mathrm{opt}}$ Gaussian distributions can be interpreted as spatial estimates for the brain regions underlying the experiments of the set.

In order to determine the optimal number of components $\mathrm{K}_{\mathrm{opt}}$, the Bayesian information criterion (BIC) is used, which is a statistical index for model selection that estimates the optimal dimensionality of the data while avoiding overfitting [15]. Thus, in PaMiNI, Gaussian mixture modeling is repetitively performed for ascending numbers of components and the mixture model is chosen, where the BIC is minimal.
In the second step of PaMiNI, the frequent co-activation patterns across experiments are identified within the modeled brain regions by an adapted association analysis approach. Association analysis originally arose from economy, i.e. market-basket analysis, where implications about the buying behavior of clients can be derived from the products that are commonly bought by customers [16]. In PaMiNI, the terms and methods from association analysis are adapted to the problem of mining co-activation patterns from neuroimaging experiments, such that the modeled brain regions correspond to items, the experiments of a set to transactions, and the co-activation patterns to frequent itemsets, which are to be identified. For each experiment, the set of modeled brain regions, featuring activations, is determined. For this, each activation focus is assigned to a modeled brain region from the first step of PaMiNI, when its probability in the corresponding Gaussian distribution of the mixture model reaches a specific threshold. In contrast to the initial publication of the PaMiNI method [9], we used here a probability threshold relative to the maximal probability of the specific distribution instead of the posterior probability. As a result, the allocation of activation foci is determined by a relative distance to the center of the distribution and cannot be distorted by adjoining distributions, when brain regions lie close to each other or overlap. Through the allocation of activation foci to the modeled brain regions, each experiment of a dataset can be represented as a subset of the $\mathrm{K}_{\text {opt }}$ Gaussian distributions, i.e. the modeled brain regions in which it provides activation foci. The frequent co-activation patterns are efficiently revealed in PaMiNI by applying the Apriori algorithm [16]. The Apriori algorithm utilizes a monotonicity property of frequent itemsets, i. e. that any subset of a frequent itemset must be frequent, and iteratively calculates frequent k-itemsets from the frequent $(\mathrm{k}-1)$-itemsets, starting with the frequent single items (1-itemsets). As a result, PaMiNI yields all combinations of brain regions showing common activation in at least a minimum number of the included studies (minimum support), that is, the frequent co-activation patterns of the dataset.

\section{Application of PaMiNI on the FG1 and FG2 experiments}

The datasets of experiments for FG1 and FG2 were analyzed separately in PaMiNI. Gaussian mixture modeling was performed for up to 20 components $\left(\mathrm{K}_{\max }\right)$ and with 5000 repetitions. Gaussian distributions were excluded from the further analysis if their standard deviation in one dimension exceeded $25 \mathrm{~mm}$. Activation foci were assigned to the modeled brain regions if they passed a probability threshold of 0.25 of the maximum probability of the distribution (probability of the mean) corresponding to a full width at quarter maximum (FWQM) diameter around the center. Co-activation patterns were considered frequent if they were found in at least 20 experiments (minimum support) and only patterns that included the seed in one hemisphere were considered. Analysis was conducted with the latest version of the PaMiNI graphical user interface [17] (http://www.fz-juelich. de/inm/inm-1/DE/Service/Download/download_node.html). Allocation to anatomical regions of JuBrain, the Jülich brain atlas, was applied using the SPM Anatomy toolbox [18].

\section{Results \\ $\nabla$}

\section{Co-activation patterns of FG1}

For the analysis of experiments with activation within FG1, the mixture model with 15 components was chosen as indicated by 
the BIC. Of these, three were excluded by exceeding the standard deviation threshold of $25 \mathrm{~mm}$, resulting in 12 clusters ( Table 1 , - Fig. 2a). Two corresponded to the bilateral seed regions on the fusiform gyrus. Two were located immediately posterior to the seeds in the early visual cortex on either hemisphere overlapping with ventral areas V3v and hV4 [19], and lateral occipital areas V4la and V4lp [20]. One cluster represented the primary visual cortex V1 [21] of both hemispheres by overlapping the interhemispheric fissure, partially reaching into V2 [21] of both sides. Two clusters were centered in the intraparietal sulcus of both sides overlapping with areas hIP1[22], hIP3 and partially with 7A [23]. Two clusters were located on the inferior frontal gyrus bilaterally overlapping with areas 44 and 45 [24], and reaching onto the middle frontal gyrus. On the right side, this cluster extended into the

Table 1 Brain regions identified by Gaussian mixture modeling in PaMiNI for the FG1 and FG2 datasets. Main location of Gaussian distribution, the threedimensional coordinates of their centers in MNI stereotaxic space, as well as their standard deviations in all three dimensions are specified.

Tab. 1 Hirnregionen, die durch Gaussian mixture modeling in PaMiNI für die Datensätze von FG1 und FG2 identifiziert wurden. Die vorwiegende Lokalisation der Gaußverteilungen, die dreidimensionalen Koordinaten ihrer Zentren im stereotaktischen MNI Referenzraum sowie ihre Standardabweichung in allen drei Raumrichtungen ist angegeben.

\begin{tabular}{|c|c|c|c|c|c|c|c|c|c|c|c|c|c|}
\hline \multicolumn{7}{|l|}{ FG1 dataset } & \multicolumn{7}{|l|}{ FG2 dataset } \\
\hline \multirow{2}{*}{$\begin{array}{l}\text { location } \\
\text { fusiform gyrus L (FG1) }\end{array}$} & \multicolumn{3}{|c|}{$\begin{array}{l}\text { MNI coordinates } \\
(\mathrm{x}, \mathrm{y}, \mathrm{z})\end{array}$} & \multicolumn{3}{|c|}{$\begin{array}{l}\text { standard deviation } \\
(\mathrm{x}, \mathrm{y}, \mathrm{z})[\mathrm{mm}]\end{array}$} & \multirow{2}{*}{$\begin{array}{l}\text { location } \\
\text { fusiform gyrus L (FG2) }\end{array}$} & \multicolumn{3}{|c|}{$\begin{array}{l}\text { MNI coordinates } \\
(x, y, z)\end{array}$} & \multicolumn{3}{|c|}{$\begin{array}{l}\text { standard deviation } \\
(\mathrm{x}, \mathrm{y}, \mathrm{z})[\mathrm{mm}]\end{array}$} \\
\hline & -36 & -68 & -13 & 7.7 & 11.8 & 5.8 & & -41 & -71 & -12 & 4.9 & 9.7 & 7.6 \\
\hline fusiform gyrus R (FG1) & 35 & -67 & -13 & 6.8 & 13.5 & 6.7 & fusiform gyrus R (FG2) & 42 & -68 & -14 & 6.2 & 11.2 & 8.0 \\
\hline primary visual & 4 & -78 & -8 & 17.8 & 13.6 & 18.5 & primary visual & 7 & -76 & -9 & 18.7 & 12.4 & 18.0 \\
\hline early visual L & -29 & -86 & -2 & 11.7 & 8.5 & 10.1 & early visual L & -24 & -92 & -3 & 10.4 & 6.5 & 9.0 \\
\hline early visual R & 37 & -79 & -1 & 11.7 & 12.6 & 11.4 & early visual R & 29 & -88 & 1 & 9.0 & 7.6 & 12.5 \\
\hline intraparietal sulcus L & -28 & -62 & 45 & 11.1 & 15.7 & 12.0 & superior occipital gyrus L & -26 & -73 & 38 & 8.2 & 10.6 & 16.2 \\
\hline intraparietal sulcus $\mathrm{R}$ & 28 & -67 & 43 & 7.7 & 13.2 & 13.3 & intraparietal sulcus L & -38 & -44 & 48 & 8.3 & 11.8 & 8.8 \\
\hline inferior frontal gyrus L & -44 & 14 & 22 & 7.4 & 18.5 & 17.0 & $\begin{array}{l}\text { superior occipital gyrus/ } \\
\text { intraparietal sulcus R }\end{array}$ & 31 & -62 & 45 & 9.0 & 12.2 & 10.5 \\
\hline inferior frontal gyrus $\mathrm{R}$ & 45 & 18 & 9 & 10.5 & 24.1 & 16.1 & superior temporal gyrus L & -54 & -41 & 10 & 6.7 & 18.5 & 17.1 \\
\hline central/ precentral R & 44 & -8 & 43 & 11.5 & 22.0 & 12.9 & superior temporal gyrus $\mathrm{R}$ & 55 & -36 & 10 & 7.8 & 17.7 & 17.1 \\
\hline $\begin{array}{l}\text { dorsal premotor/ supple- } \\
\text { mentary motor area L }\end{array}$ & -14 & 1 & 58 & 17.8 & 9.6 & 5.0 & inferior frontal gyrus L & -46 & 19 & 13 & 8.4 & 19.3 & 16.5 \\
\hline \multirow[t]{6}{*}{ superior temporal gyrus L } & -54 & -35 & 9 & 6.5 & 19.5 & 16.7 & inferior frontal gyrus R & 43 & 25 & 2 & 9.4 & 17.7 & 17.1 \\
\hline & & & & & & & anterior insula L & -34 & 22 & -1 & 3.7 & 6.1 & 5.8 \\
\hline & & & & & & & premotor L & -39 & -3 & 45 & 11.1 & 9.7 & 13.4 \\
\hline & & & & & & & premotor R & 43 & 3 & 40 & 11.4 & 15.3 & 14.8 \\
\hline & & & & & & & $\begin{array}{l}\text { supplementary } \\
\text { motor area }\end{array}$ & 0 & 13 & 50 & 6.0 & 9.2 & 8.4 \\
\hline & & & & & & & thalamus L & -9 & -17 & 1 & 11.6 & 13.3 & 11.0 \\
\hline
\end{tabular}

L: left hemisphere; R: right hemisphere.

L: linke Hemisphäre; R: rechte Hemisphäre.
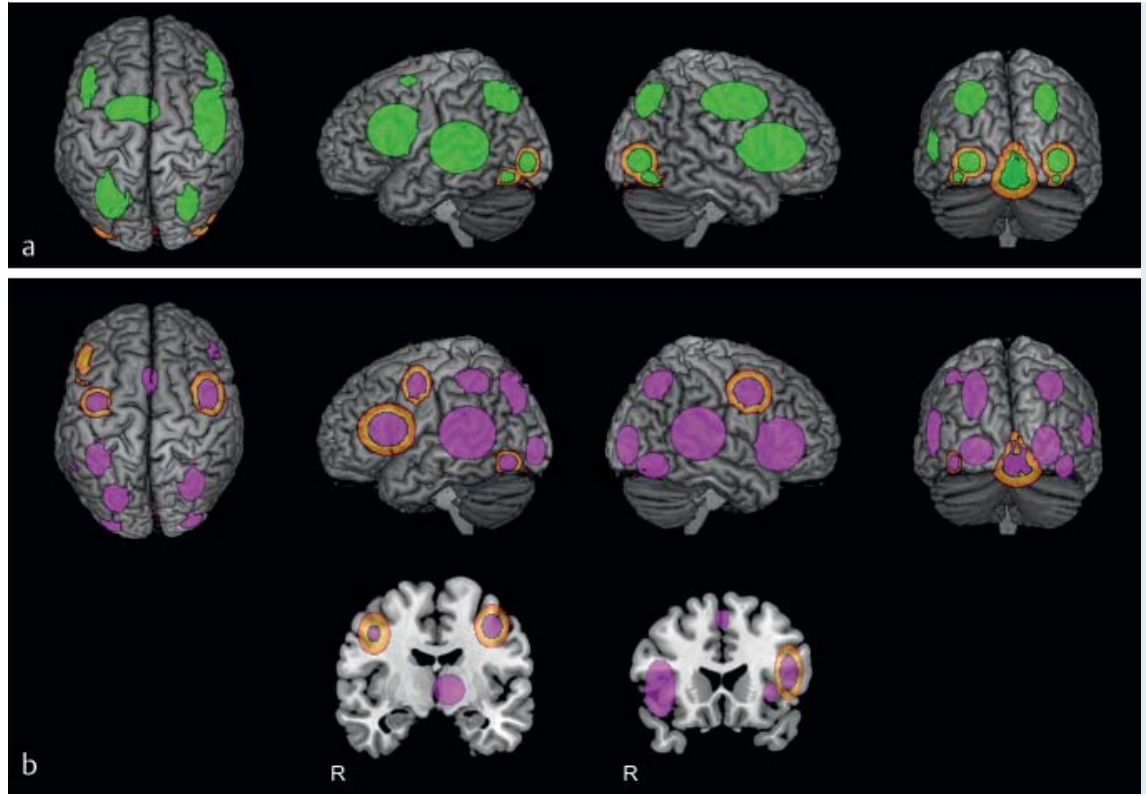

Fig. 2 Modeled brain regions and most frequent 5-pattern of the FG1 (a, green) and FG2 (b, violet) datasets. Gaussian distributions (restricted to 1FWHM) resulting from Gaussian mixture modeling in PaMiNI are projected onto the MNI single subject reference template. Top view, left view, right view and back view are shown (from left to right). For FG2 (b), two coronal sections are shown additionally. The regions belonging to the most frequent 5pattern of each dataset are highlighted in orange.

Abb. 2 Modellierte Hirnregionen und häufigstes 5er-Muster der FG1 (a, grün) und FG2 (b, violett) Datensätze. Die aus dem Gaussian mixture modeling in PaMiNI hervorgehenden Gauß-Verteilungen (beschränkt auf 1FWHM) sind auf das MNI Einzelprobanden-Referenzgehirn projiziert. Ansichten von oben, von links, von rechts und von hinten sind abgebildet (von links nach rechts). Für FG2 (b) sind zusätzlich zwei koronare Schnitte abgebildet. Die Regionen, die zum jeweils häufigsten 5er-Muster des Datensatzes gehören, sind in orange hervorgehoben. 
Table 2 Frequent co-activations (2-patterns) of FG1 and FG2. Co-active brain regions and the number of experiments, in which co-activation was found, are listed with decreasing frequency for each side of FG1 and FG2 separately.

Tab. 2 Häufige Koaktivierungen (2er-Muster) von FG1 und FG2. Koaktivierte Hirnregionen und die Anzahl der Experimente, in denen die Koaktivierung gefunden wurde, sind mit absteigender Häufigkeit aufgelistet.

\begin{tabular}{|c|c|c|c|c|c|c|c|}
\hline left FG1 & & right FG1 & & left FG2 & & right FG2 & \\
\hline early visual L & 90 & early visual $\mathrm{R}$ & 94 & inferior frontal gyrus L & 115 & FG2 L & 107 \\
\hline early visual R & 79 & FG1 L & 73 & FG2 R & 107 & primary visual & 90 \\
\hline FG1 R & 73 & primary visual & 68 & primary visual & 102 & inferior frontal gyrus L & 87 \\
\hline primary visual & 68 & early visual L & 68 & early visual $R$ & 88 & intraparietal sulcus $R$ & 67 \\
\hline inferior frontal gyrus $\mathrm{R}$ & 54 & intraparietal sulcus $R$ & 51 & premotor $\mathrm{R}$ & 86 & early visual $R$ & 65 \\
\hline inferior frontal gyrus $L$ & 49 & inferior frontal gyrus $R$ & 50 & inferior frontal gyrus $R$ & 81 & premotor $\mathrm{R}$ & 64 \\
\hline central/precentral R & 47 & inferior frontal gyrus L & 47 & premotor L & 80 & inferior frontal gyrus $R$ & 63 \\
\hline intraparietal sulcus $R$ & 45 & central/precentral R & 45 & intraparietal sulcus $R$ & 73 & thalamus L & 57 \\
\hline Intraparietal sulcus L & 44 & intraparietal sulcus L & 40 & early visual L & 71 & early Visual L & 56 \\
\hline superior temporal gyrus L & 31 & $\begin{array}{l}\text { premotor/ supplementary } \\
\text { motor area L }\end{array}$ & 32 & intraparietal sulcus L & 64 & premotor L & 56 \\
\hline $\begin{array}{l}\text { premotor/ supplementary } \\
\text { motor area L }\end{array}$ & 31 & superior temporal gyrus L & 28 & superior temporal gyrus L & 62 & superior occipital gyrus L & 52 \\
\hline \multirow[t]{5}{*}{ superior temporal } & & & & supplementary motor area & 60 & superior temporal gyrus L & 51 \\
\hline & & & & superior temporal gyrus $\mathrm{R}$ & 58 & superior temporal gyrus $\mathrm{R}$ & 50 \\
\hline & & & & thalamus L & 56 & supplementary motor area & 48 \\
\hline & & & & superior occipital gyrus L & 54 & intraparietal sulcus L & 47 \\
\hline & & & & anterior insula L & 30 & & \\
\hline
\end{tabular}

L: left hemisphere; R: right hemisphere.

L: linke Hemisphäre; R: rechte Hemisphäre.

left anterior insula. One cluster was located at the central and precentral region of the right hemisphere, overlapping with parts of areas $3 \mathrm{~b}$ [25], 4a [26] and 6. There was one component at the dorsal premotor cortex of the left hemisphere in the region of the supplementary motor area. Finally, one cluster was centered at the superior temporal gyrus of the left hemisphere reaching into area PFcm of the inferior parietal lobule [27] dorsally and the middle temporal gyrus ventrally.

All co-activations (2-patterns) with FG1 are listed in 0 Table 2. The most frequent co-activations of FG1 were with areas within the visual cortex. In particular, for both hemispheres, FG1 was most commonly co-active with the ipsilateral early visual cortex in 94 experiments (right FG1) and 90 experiments (left FG1), followed by co-activations with the contralateral early visual cortex, the primary visual cortex and the contralateral FG1, which occurred in 68 to 79 experiments. Co-activations with extra-visual areas were much rarer. Here, the bilateral inferior frontal gyrus was under the most commonly co-activated extra-visual regions for both sides of the seed with up to 50 experiments. The frequent higherdimensional co-activation patterns also mainly consisted of visual areas. Characteristically, the most frequent 5-pattern found in 21 experiments consisted of the bilateral FG1, the bilateral early visual cortex and the primary visual representation ( $\bullet$ Fig. $2 a$ ).

\section{Co-activation patterns of FG2}

For FG2, the PaMiNI analysis yielded a mixture model with 21 components based on the minimal BIC. Of these, 4 regions were excluded due to their standard deviation exceeding the threshold, resulting in 17 regions for the further analysis ( $\bullet$ Table 1, - Fig. 2b). Two regions corresponded to the bilateral seeds on the fusiform gyrus. Similar to the analysis of FG1, two clusters were located bilaterally in the early visual cortex posterior to the seed overlapping with areas V3v, hV4 [19] and V4lp [20], and one cluster represented the primary and partially secondary visual cortex V1 and V2 [21] of both sides. On the right hemi- sphere one cluster was located on the superior occipital gyrus and intraparietal sulcus overlapping with hIP3 [23]. On the left side, there were two separate clusters in this position, one at the superior occipital gyrus and one located in the intraparietal sulcus overlapping with hIP1 [22], hIP3 and partially with 7PC [23]. Two regions were centered on the bilateral superior temporal gyrus, both partially reaching the middle temporal gyrus and areas PFm and PFcm [27] of the inferior parietal lobule. Two clusters were found on the inferior frontal gyrus bilaterally, overlapping with areas 44 and 45 [24]. On the right side, this cluster extended into the anterior insula. On the left hemisphere, there was an additional cluster representing the left anterior insula. Two regions were located bilaterally in the premotor cortex and one cluster corresponded to the SMA of both hemispheres. Finally, one cluster was located in the left thalamus.

The frequent co-activations with FG2 are listed in $\bullet$ Table 2. Compared to FG1, FG2 was more commonly co-active with extra-visual areas. Furthermore, while frequencies of co-activations were quite symmetrical for FG1, the left FG2 co-activations were more common than the right-sided ones. The most frequent co-activation existed between left FG2 and the left inferior frontal gyrus found in 115 experiments. This was followed by the bilateral activation of FG2 in 107 experiments and co-activations with the primary visual cortex in 102 experiments for left FG2 and 90 experiments for right FG2. For right FG2, the co-activation with left inferior frontal gyrus was only found in 87 experiments. Co-activations with the early visual cortex were similarly often found as co-activations with several extra-visual areas, where the right inferior frontal gyrus, the bilateral premotor region and the bilateral intraparietal sulcus were most commonly co-active (between 63 and 87 times). The higher-dimensional co-activation patterns also mainly showed extra-visual connections and a dominance of left FG2, e.g. the most frequent 3-pattern found in 60 experiments consisted of left FG2, the left inferior frontal gyrus and the left premotor region. Adding the right premotor region to 
this pattern resulted in the most frequent 4-pattern, still found in 40 experiments. The most frequent 5-pattern, found in 26 experiments, condensed the frequent co-activations of the FG2 set. It consisted of left FG2, the left inferior frontal gyrus, the bilateral premotor region as well as the primary visual cortex ( $\bullet$ Fig. 2 b).

\section{Discussion}

$\nabla$

In the current study, we revealed differential co-activation patterns of ventral visual areas FG1 and FG2 using the recent metaanalytic technique PaMiNI. The analysis in PaMiNI showed that co-activations of FG1 are frequently found within the visual system and are quite symmetrically distributed across both hemispheres, while FG2 is more co-active with extra-visual areas and shows a dominance of the left side.

The current results are well in line with a previous analysis of the task-based functional connectivity of FG1 and FG2 using metaanalytic connectivity modeling (MACM) [6]. Except for some deviations, the Gaussian mixture modelling in PaMiNI yielded quite similar regions for the FG1 and FG2 datasets. These regions largely correspond to the network of common connections to FG1 and FG2 from the MACM analysis. Furthermore, large parts of this network, i.e. the intraparietal sulcus, inferior frontal gyrus, premotor region, supplementary motor area and anterior insula, correspond to the "task-positive-network", which is thought to be generally active during cognitive tasks demanding targeted attention [28]. The involvement of FG1 and FG2 in this network, which has now been consistently demonstrated by two independent approaches, thus hints at the role of both areas in visual object recognition requiring goal-directed focusing and integration of visual object representations into other cortical systems.

Beyond such correspondence between the two approaches, the PaMiNI distribution of co-activation patterns further points to differential hierarchical roles for FG1 and FG2. This is important, as both areas are located at the junction between the early ventral visual cortex and the higher-order visual areas [4]. The prevalence of co-activations with visual areas as well as the quite symmetric distribution of co-activations for FG1 is characteristic for an early visual area. Considering the connections of FG1 to areas of other functional systems and evidence from previous analyses of the functional involvement of this area [6], we suggest that FG1 occupies an intermediate hierarchical level between the early visual areas and the higher-order ones. In contrast, the more common extra-visual co-activations of FG2 and the clear lateralization strongly indicate that FG2 is a higher-order visual area. Hence, FG1 probably facilitates more basic functions in visual processing for object recognition like extraction of simple shapes, while FG2 is directly involved in associative, object-specific recognition. As previously shown, FG2 probably corresponds to the posterior part of the fusiform face area $[4,6,7]$, which is essential for the recognition and identification of faces. Furthermore, FG2 was shown to be involved in the visual processing of written language [6] and is located in proximity to the visual word-form area [8]. The frequent co-activation of left FG2 with the left inferior frontal gyrus probably represents an important connection of FG2 to the language network in its function for reading [29].

The functional roles for FG1 and FG2 indicated by the current analysis are well in line with the hierarchical segregation of both areas previously revealed at the neurochemical level, i. e. receptor architecture [5]. Here, the analysis of 15 neurotransmitter-recep- tor concentrations yielded a similarity of the receptor profile of FG2 with that of associative parietal areas, while FG1 was similar but yet distinct to early visual areas.

It should be noted that the revealed co-activation patterns rely on existing fMRI experiments, which by nature yield an indirect measure of brain activity and entail several methodological limitations, particularly when comparing between experiments [11]. As a consequence, the co-activation patterns are only an indirect indicator of brain connectivity and all conclusions have to be drawn under consideration of this background. Nevertheless, co-activations derived from coordinate-based meta-analyses have been shown to be a suitable surrogate for functional connectivity and show good correspondence with resting-state connectivity [11].

The PaMiNI method used for the current study is a novel metaanalytic approach for the detection of co-activation patterns based on pattern-mining techniques. In contrast to previous meta-analytic approaches like MACM [30], it considers real within-experiment co-activation instead of convergence of activation foci across all experiments of a set [9] and allows for investigations of the subnetwork structure of functionally connected regions. Compared to our first presentation of PaMiNI [9], we analyzed here co-activations to a specific seed region instead of thematically selected datasets. Thus, the current study expands the spectrum of PaMiNI from conventional coordinate-based meta-analysis to seed-related co-activation mapping and further emphasizes the applicability of this approach.

\section{Conclusion}

\section{$\nabla$}

We revealed differential co-activation patterns of the ventral visual areas FG1 and FG2 on the fusiform gyrus that elucidate their hierarchical level in the ventral visual processing stream. FG1 shows features of an intermediate area for object recognition with mainly intra-visual co-activity, while FG2 is a categoryspecific higher order area showing diverse extra-visual co-activations. Hence, the transition between the early and the higher-order ventral visual cortex can be determined at the border between both areas on the posterior fusiform gyrus, i.e. in the mid-fusiform sulcus [7], which clearly improves our understanding of the organization of the object-related visual system in humans.

\section{Clinical relevance}

- The transition between early and higher-order ventral visual cortex can be found between areas FG1 and FG2 at the posterior mid-fusiform sulcus, yielding a helpful landmark for structure-function relationships.

- The differential roles of areas FG1 and FG2 located on the medial and lateral bank of the fusiform gyrus yield possible implications for deficits of occipitotemporal lesions, e.g. following cerebral ischemia.

- The applicability of the PaMiNI approach for the analysis of co-activation patterns and their functional implications is further substantiated, which emphasizes the convenient use for functional brain-network investigations. 


\section{References}

1 Grill-Spector K, Malach R. The human visual cortex. Annu Rev Neurosci 2004; 27: 649-677

2 Wandell BA, Winawer J. Imaging retinotopic maps in the human brain. Vision Res 2011; 51: 718-737

3 Grill-Spector K, Weiner KS. The functional architecture of the ventral temporal cortex and its role in categorization. Nat Rev Neurosci 2014; 15: $536-548$

4 Caspers J, Zilles K, Eickhoff SB et al. Cytoarchitectonical analysis and probabilistic mapping of two extrastriate areas of the human posterior fusiform gyrus. Brain Struct Funct 2013; 218: $511-526$

5 Caspers J, Palomero-Gallagher N, Caspers $S$ et al. Receptor architecture of visual areas in the face and word-form recognition region of the posterior fusiform gyrus. Brain Struct Funct 2015; 220: 205-219

6 Caspers J, Zilles $K$, Amunts $K$ et al. Functional characterization and differential coactivation patterns of two cytoarchitectonic visual areas on the human posterior fusiform gyrus. Hum Brain Mapp 2014; 35 : $2754-2767$

7 Weiner KS, Golarai G, Caspers J et al. The mid-fusiform sulcus: a landmark identifying both cytoarchitectonic and functional divisions of human ventral temporal cortex. Neuroimage 2014; 84: 453-465

8 Cohen L, Dehaene S, Naccache L et al. The visual word form area: spatial and temporal characterization of an initial stage of reading in normal subjects and posterior split-brain patients. Brain 2000; 123: 291 - 307

9 Caspers J, Zilles K, Beierle C et al. A novel meta-analytic approach: mining frequent co-activation patterns in neuroimaging databases. Neuroimage 2014; 90: 390-402

10 Laird AR, Lancaster JL, Fox PT. BrainMap: the social evolution of a human brain mapping database. Neuroinformatics 2005; 3: 65-78

11 Eickhoff SB, Grefkes C. Approaches for the integrated analysis of structure, function and connectivity of the human brain. Clin EEG Neurosci 2011; 42: 107-121

12 Felleman DJ, van Essen DC. Distributed hierarchical processing in the primate cerebral cortex. Cereb Cortex 1991; 1: 1-47

13 Caspers J, Zilles K, Eickhoff SB et al. Coordinate-Based Pattern-Mining on Functional Neuroimaging Databases. In: Greco S, Bouchon-Meunier B, Coletti $\mathrm{G}$ et al. eds Advances on Computational Intelligence. Berlin Heidelberg: Springer; 2012: 240-249

14 Dempster AP, Laird NM, Rubin DB. Maximum likelihood from incomplete data via the EM algorithm. Journal of the Royal Statistical Society Series B (Methodological) 1977; 39: 1 - 38

15 Schwarz G. Estimating the dimension of a model. The annals of statistics 1978; 6: $461-464$
16 Agrawal $R$, Srikant $R$. Fast algorithms for mining association rules. In: Proc 20th Int Conf Very Large Data Bases, VLDB. 1994: 487-499

17 Caspers J,Zilles K, Eickhoff SB et al. PaMiNI: A comprehensive system for mining frequent neuronal patterns of the human brain. In: 25th International Symposium on Computer-Based Medical Systems (CBMS), 2012. 2012

18 Eickhoff SB, Paus T, Caspers S et al. Assignment of functional activations to probabilistic cytoarchitectonic areas revisited. Neuroimage 2007; 36: $511-521$

19 Rottschy C, Eickhoff SB, Schleicher A et al. Ventral visual cortex in humans: cytoarchitectonic mapping of two extrastriate areas. Hum Brain Mapp 2007; 28: 1045 - 1059

20 Malikovic A, Amunts K, Schleicher A et al. Cytoarchitecture of the human lateral occipital cortex: mapping of two extrastriate areas hOc4la and hOc4lp. Brain Struct Funct 2015

21 Amunts K, Malikovic A, Mohlberg $\mathrm{H}$ et al. Brodmann's areas 17 and 18 brought into stereotaxic space-where and how variable? Neuroimage 2000; 11 : $66-84$

22 Choi HJ, Zilles K, Mohlberg $\mathrm{H}$ et al. Cytoarchitectonic identification and probabilistic mapping of two distinct areas within the anterior ventral bank of the human intraparietal sulcus. J Comp Neurol 2006; 495: 53 69

23 Scheperjans F, Eickhoff SB, Homke L et al. Probabilistic maps, morphometry, and variability of cytoarchitectonic areas in the human superior parietal cortex. Cereb Cortex 2008; 18: 2141 - 2157

24 Amunts $K$, Schleicher A, Burgel $U$ et al. Broca's region revisited: cytoarchitecture and intersubject variability. J Comp Neurol 1999; 412: $319-341$

25 Geyer S, Schormann T, Mohlberg $H$ et al. Areas 3a, 3b, and 1 of human primary somatosensory cortex. Part 2. Spatial normalization to standard anatomical space. Neuroimage 2000; 11: 684-696

26 Geyer S, Ledberg A, Schleicher A et al. Two different areas within the primary motor cortex of man. Nature 1996; 382: 805-807

27 Caspers S, Eickhoff SB, Geyer S et al. The human inferior parietal lobule in stereotaxic space. Brain Struct Funct 2008; 212: 481 - 495

28 Fox MD, Snyder AZ, Vincent JL et al. The human brain is intrinsically organized into dynamic, anticorrelated functional networks. Proc Natl Acad Sci U S A 2005; 102: 9673-9678

29 Price CJ. The Functional Anatomy of Reading. In: Richard SJF, Karl JF, Christopher DF et al. eds Human Brain Function (Second Edition). Burlington: Academic Press; 2004: 547-562

30 Robinson JL, Laird AR, Glahn DC et al. Metaanalytic connectivity modeling: delineating the functional connectivity of the human amygdala. Hum Brain Mapp 2010; 31: 173-184 